\title{
Device for robotic picking of strawberries
}

\author{
D. O. Khort ${ }^{1}$, A. I. Kutyrev ${ }^{1,}$, , R. A. Filippov ${ }^{1}$, and R. V. Vershinin ${ }^{1}$ \\ ${ }^{1}$ Federal Scientific Agroengineering Center VIM, 1-st Institutsky proezd, 5, Moscow, 109428, Russia
}

\begin{abstract}
The article presents a device for collecting strawberry berries, presents a 3D model of an automated manipulator with a computer vision system. The overall dimensions of the manipulator $(\mathrm{WxDxH}), \mathrm{mm}$ $200 \times 700 \times 600$, the number of degrees of freedom - 6, load capacity $-1 \mathrm{~kg}$, the maximum effort to detach fruits and berries - 10-80 N. An algorithm for the operation of a computer vision system for determining the boundaries of berries and their ripeness based on size factor. The results of field experiments, measuring the area of berries of wild strawberries at different times of the day are given. It was established that the lighting conditions have a significant effect on the detection of the boundaries and area of garden strawberries, and their ripeness.
\end{abstract}

\section{Introduction}

Currently, scientists are conducting research to assess the feasibility of using automated devices with intelligent work algorithms in industrial gardening, which will allow the implementation of intelligent agricultural technologies in strawberry stands at a new level $[1,2]$. The main component of successful automated harvesting is the ability to process sensory data, evaluate and understand the input video data. Computer vision systems in horticulture are used to count the number of fruits and berries, monitor the condition of plants, control pests and diseases, topographic analysis of plantings, control various parameters of the soil and the environment [3,4]. The most important tasks when performing automated harvesting of berry and fruit crops in the field is to accurately determine the boundaries of fruits and berries, as well as to assess the degree of ripeness using a computer vision camera under various lighting conditions [5-7]. The purpose of the study was to develop a 3D model of an automated manipulator and an algorithm for recognizing location coordinates based on size and ripeness factors using the example of wild strawberry berries under various lighting conditions.

\section{Materials and methods}

The 3D model of the automated manipulator was designed in CAD "Compass-3D v17" using methods of mathematical modeling, theoretical mechanics and optimal design. Basler acA1920-155uc camera with GigE interface, Sony IMX174 CMOS-matrix with a frequency of 164 frames per second and computer vision library Open Source Computer

* Corresponding author: alexeykutyrev@gmail.com 
Vision Library (OpenCV) were used to recognize ripeness of berries by color tone and their coordinates at a distance of 30 to $200 \mathrm{~mm}$. The camera matrix has a resolution of up to 1920 x 1200 pixels, a resolution of 2.3 megapixels (interpolated). For conducting experiments, measuring illumination, a Radex Lupin luxmeter (Quarta Rad, Russia) was used, the relative measurement error of $10 \%$. Program code and algorithm for determining the coordinates of the berry, the degree of maturity and the calculation of the distance to the berry developed in the text editor Sublime Text 3 in the programming language Python 3.7.2.

\section{Results and discussions}

As a result of the research, a model of an automated manipulator was developed and visualized (Fig. 1).

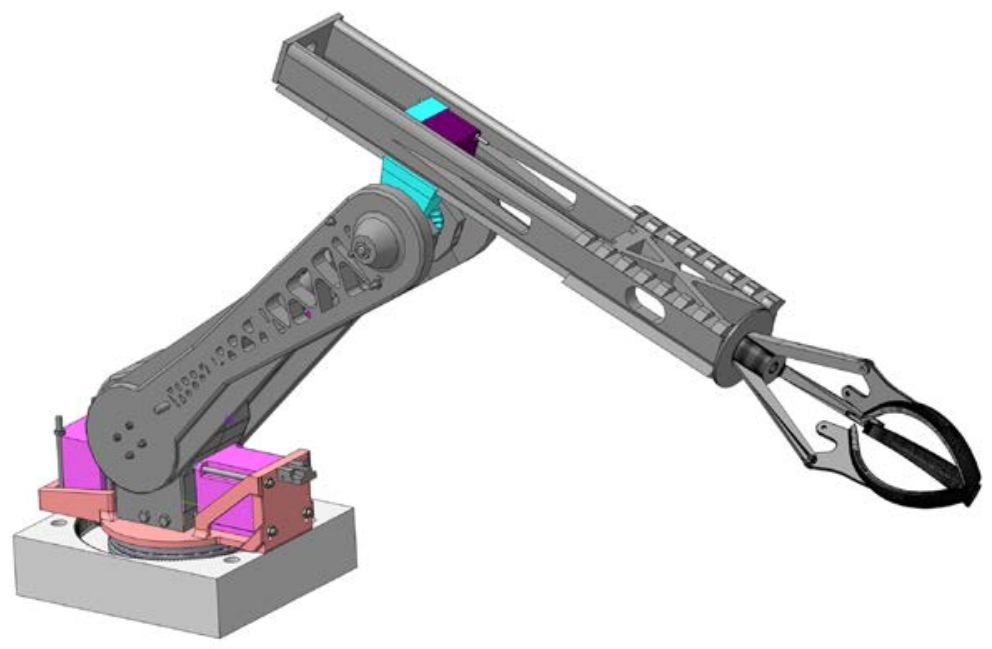

Fig. 1. 3D model of an automated manipulator

Initially, to calculate the technological parameters of the manipulator, there was a number of input data: angular velocity, angle of rotation of the link, mass of individual links, the parameters of the output data were justified: the coordinates of the location of the links in space, forces, moments, angular accelerations acting in the joints of the links (Fig.2).

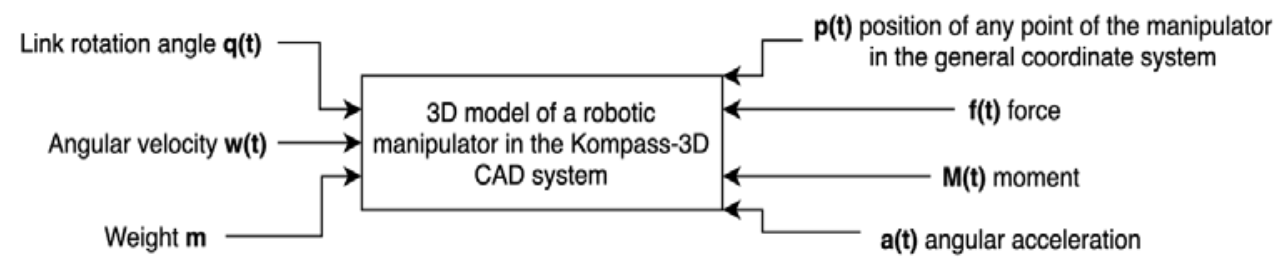

Fig. 2. Input and output data in the manipulator model

The overall dimensions of the manipulator $(\mathrm{WxDxH}), \mathrm{mm}-200 \times 700 x 600$, the number of degrees of freedom - 6, load capacity - $1 \mathrm{~kg}$, the maximum separation force of fruits and berries - 10-80 N. To determine the working area of the manipulator, the manipulator was gripped over the entire range of permissible angles. Based on the coordinates of the contour 
points, a working zone is constructed on the coordinate plane. The contour of the working area is shown on a plane along the axes OXZ and OXY (Fig. 3).
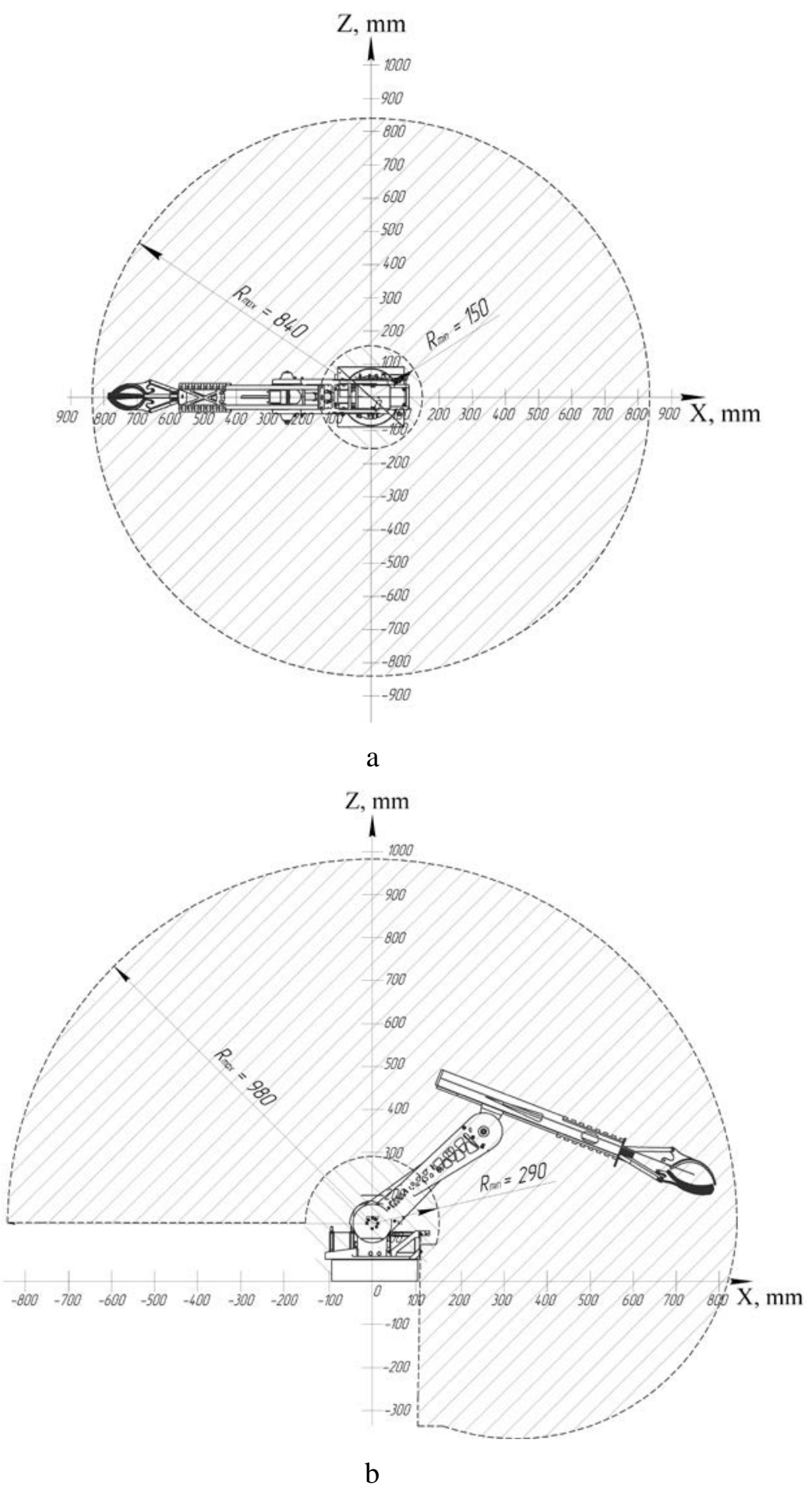

Fig. 3. The contour of the working area of the manipulator model: a - side view, b - top view 
The use of stepper motors Nema 343HS5628, Nema 23HS5628, providing the capture of berries in a given working area of coverage of the manipulator with a given speed, is justified as a drive of the links of the manipulator (Table 1).

Table 1. Technical characteristics of the manipulator

\begin{tabular}{|c|c|}
\hline Indicator & Value \\
\hline Weight of the manipulator (kg) & 11.5 \\
\hline Vertical reach (mm) & 980 \\
\hline Horizontal reach (mm) & 840 \\
\hline Voltage (V) & 12 \\
\hline $\begin{array}{c}\text { The radius of the coverage of the } \\
\text { working area (mm) }\end{array}$ & 720 \\
\hline Payload (kg) & 2.5 \\
\hline Movement Accuracy (+/-) & 1.5 \\
\hline
\end{tabular}

In the programming language Python 3.7.2, an algorithm has been developed and implemented that includes operations to determine the coordinates of a berry or fruit, the degree of maturity, and calculate the distance to the berry or fruit. Using a computer vision camera, streaming video is captured and transferred via USB 3.0. The resulting video frames are saved and processed in the PC RAM. For each recognized strawberry or pome fruit or stone fruit, the coordinates of the $\mathrm{X}, \mathrm{Y}$, and $\mathrm{Z}$ axes are calculated. The coordinates are entered in an integer array for further processing. To recognize the degree of ripeness of strawberries, size factor was used. The size factor allows you to change the resolution obtained from the camera, which makes it possible to adjust the image detail and increase the speed of the implemented computer vision algorithm. To calibrate colors in the HSV palette (Hue, Saturation, Value - hue, saturation, value), a function for displaying color in coordinates is implemented. The red color in the HSV palette is located at the borders of two color tones $\mathrm{H}=5 / 6$ and $\mathrm{H}=1 / 6$. To determine the degree of ripeness of wild strawberries by color tone, the color ranges of ripe and unripe berries are indicated (Fig. 4).
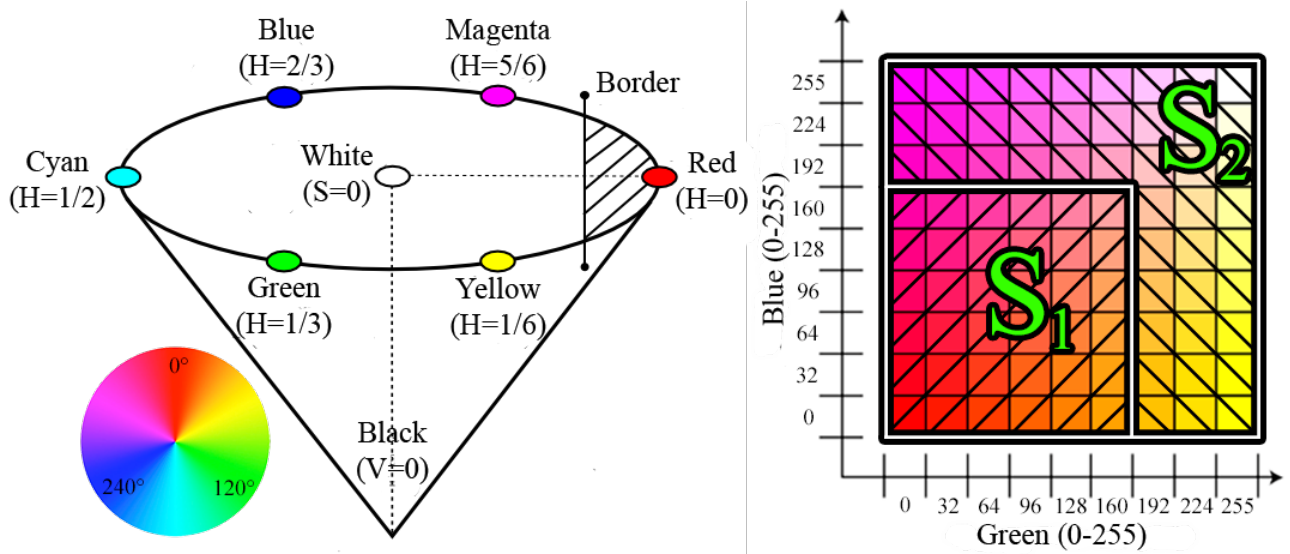

Fig. 4. The selection of the color range to determine the degree of ripeness of strawberries 
After specifying the color ranges, a mask is created for overlaying on the images. When determining the degree of ripeness of the berry due to the high pixel density and high ISO sensitivity, noise appears on the images (randomly scattered pixels of random color and brightness that do not correspond to the registered light). To level them to the image, Morphological Transformations of the OpenCV library of open and closed type were used. A normally open filter allows you to remove noise from the image, while a normally closed filter removes holes in recognized objects. After creating filters, masks are superimposed on the main image and remove unnecessary colors, leaving only the established ranges of color tones that correspond to ripe strawberries. To smooth the contour, the contour blur function is applied. From all the found contours, an array is created containing the contour itself and its coordinates. In the loop for each contour, its coordinates $\mathrm{X}, \mathrm{Y}$ and $\mathrm{Z}$ are determined, after which the algorithm proceeds to the next found berry and the cycle repeats until ripe berries remain in the collection area. A field experiment was conducted to set up operating modes and software, verify calculated parameters. A tripod with a Basler acA1920-155uc camera (Fig. 5) is installed on an industrial plantation in a row of strawberry garden.

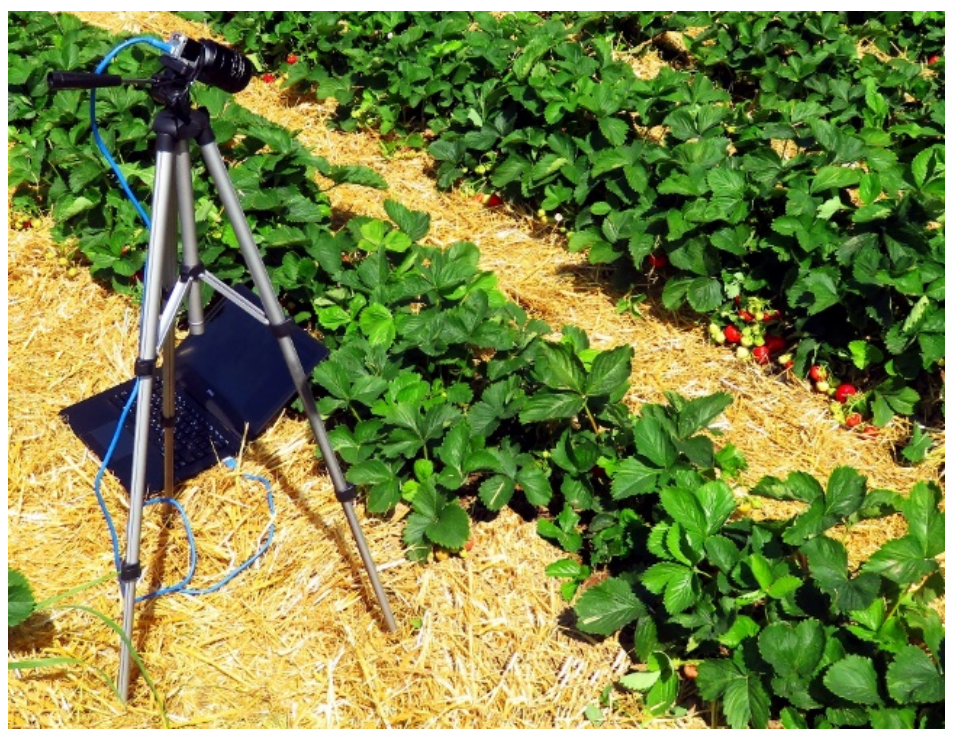

Fig. 5. Tripod with Basler acA1920-155uc camera mounted in a row of strawberries

In different lighting conditions, at different times of the day (from 9:00 a.m. to 6:00 p.m.), using the developed algorithm, we measured the area of berries in a row. Using the developed algorithm of the computer vision system, 6,000 images were processed (Fig. 6). 


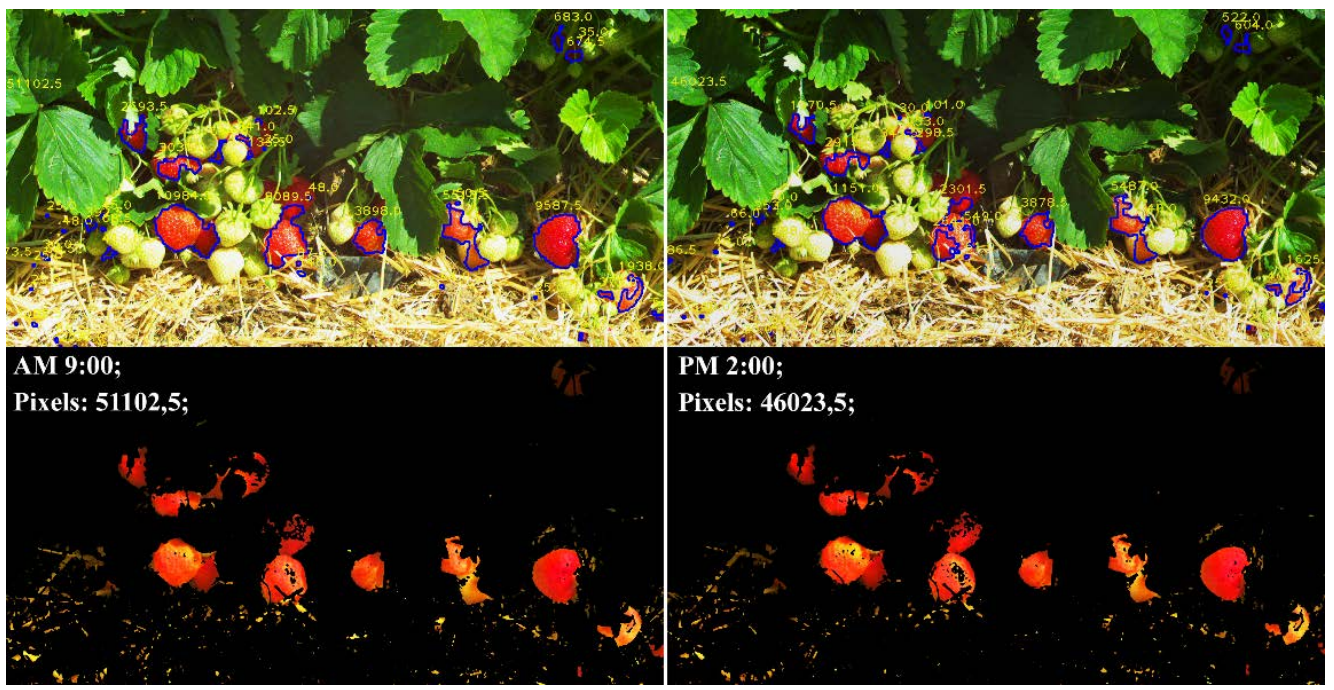

Fig. 6. Results of image processing

The area of berries for the triplicate experiment is determined in pixels (elements of a two-dimensional digital image). The found area, knowing the focal length of the camera, is converted to square centimeters. The results of the experiment are presented in table 2.

Table 2. The results of the experiment to determine the area of berries in the row of strawberries

\begin{tabular}{|c|c|c|c|c|c|c|}
\hline \multirow{2}{*}{ Indicators } & \multicolumn{7}{|c|}{$\begin{array}{c}\text { The found area of strawberry berries in various } \\
\text { lighting conditions, at different times of the day } \\
\left(\mathrm{cm}^{2}\right)\end{array}$} \\
\cline { 2 - 8 } & $9: 00$ & $10: 00$ & $12: 00$ & $14: 00$ & $16: 00$ & $18: 00$ \\
\hline Measurement 1 & 106.4 & 115.5 & 129.2 & 95.8 & 92.1 & 81.5 \\
\hline Measurement 2 & 105.6 & 115.3 & 130.1 & 95.9 & 91.6 & 80.2 \\
\hline Measurement 3 & 107.1 & 114.9 & 128.9 & 94.6 & 92.5 & 81.3 \\
\hline Average value $\left(\mathrm{cm}^{2}\right)$ & 106.4 & 115.2 & 129.4 & 95.4 & 92.1 & 81.0 \\
\hline Standard deviation, cm & 0.51 & 0.22 & 0.47 & 0.56 & 0.31 & 0.53 \\
\hline
\end{tabular}

Statistical processing of the obtained experimental data showed that the berries of wild strawberries can be distinguished from bushes (background) using their color characteristics. It was found that successful segmentation of berries and background significantly depends on climatic conditions and lighting (Fig. 7). 


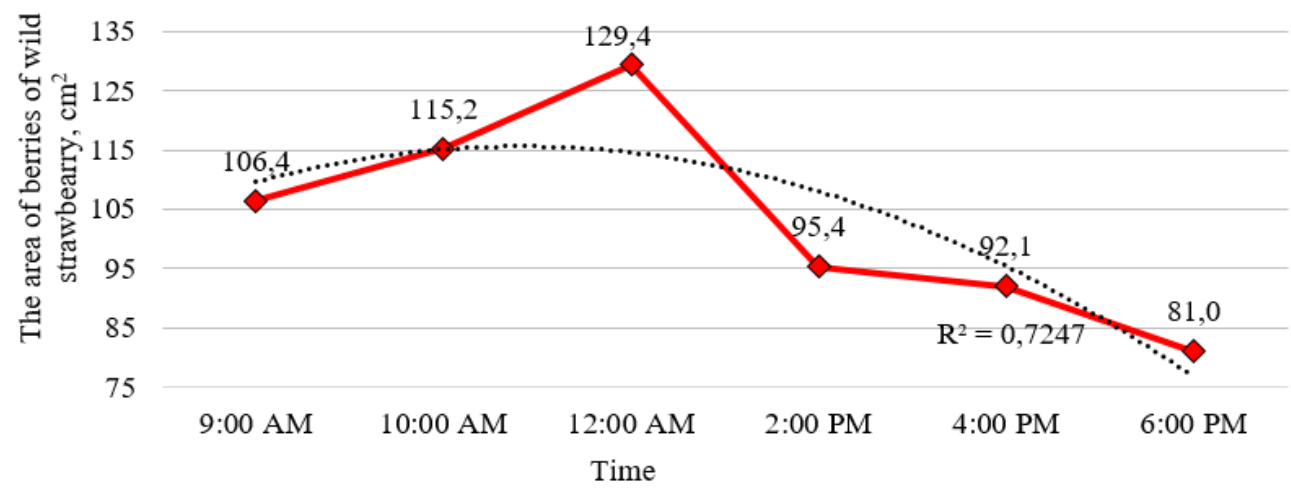

Fig. 7. A graph of the determination of the area of berries on the speed of the robotic platform and lighting conditions

The variation range of the obtained experimental data on the area of berries from 9:00 to 18:00 was $48.4 \mathrm{~cm}^{2}$. The detection efficiency of berries of their area and borders using the Basler acA1920-155uc camera and the OpenCV library with an illumination of 72,600 lux reaches $95.6 \%$. The discovered area of berries at $12: 00$ was $129.4 \mathrm{~cm}^{2}$ with a real area of berries of $135.3 \mathrm{~cm}^{2}$ (out of 64731 pixels, strawberries were detected by the garden camera in 62003 pixels). With a decrease in illumination to $56300 \mathrm{LC}$ at 18:00, the average area of the found berries decreased to $81 \mathrm{~cm}^{2}$ by $62.6 \%$ (out of 64731 pixels, strawberries were found by the garden camera to 39141.5 pixels).

\section{Conclusions}

As a result of the research, a 3D model of a device for collecting strawberries was developed. The developed manipulator will be able to function stably in conditions of industrial planting during its installation, for example on an electric drive platform. The modular design of the manipulator allows the use of various types of capture devices for its use in berry and fruit orchards. Experimental studies have shown that the developed device with a computer vision system and an algorithm for processing input video data, searching for garden strawberry berries and determining their degree of ripeness are workable, they allow strawberry berries to be recognized with a high degree of accuracy. It was established that the lighting conditions have a significant impact on the detection of strawberry berries, their area and borders, which ultimately affects the efficiency of harvesting strawberry berries in an automated mode.

\section{References}

1. Hayashi S, Shigematsu K, Yamamoto S, Kobayashi K, Kohno Y, Kamata J and Kurita M 2010 Evaluation of a strawberry-harvesting robot in a field test Biosyst. Eng 105 pp 160-171.

2. Xiong Y, Peng C, Grimstad L, From P J and Volkan Isler 2019 Development and field evaluation of a strawberry harvesting robot with acable-driven gripper Computers and Electronics in Agriculture 157 pp 392-40.

3. Dimeas F, Sako D V, Moulianitis V C and Aspragathos N A 2015 Design and fuzzy control of a robotic gripper for efficient strawberry harvesting Robotica 33 pp 10851098. More references

4. Yamamoto S, Hayashi S, Yoshida H and Kobayashi K 2014 Development of a 
stationary robotic strawberry harvester with picking mechanism that approaches target fruit from below Japan Agric. Res. Q. JARQ 48 pp 261-269.

5. Arima S, Monta M, Namba K, Yoshida Y and Kondo N 2003 Harvesting robot for strawberry grown on table top culture Journal of Society of High Technology in Agriculture 15(3) pp 162-168.

6. Cui Y, Nagata M, Guo F, Hiyoshi K, Kinoshita O and Mitarai M 2007 Study on strawberry harvesting robot using machine vision for strawberry grown on annual hill top (Part 2). Journal of Japanese Society of Agricultural Machinery 69(2) pp 60-68.

7. Mu L, Cui G, Liu Y, Cui Y, Fu L and Gejima Y 2019 Design and simulation of an integrated end-effector for picking kiwifruit by robot Information Processing in Agriculture doi:10.1016/j.inpa.2019.05.004 